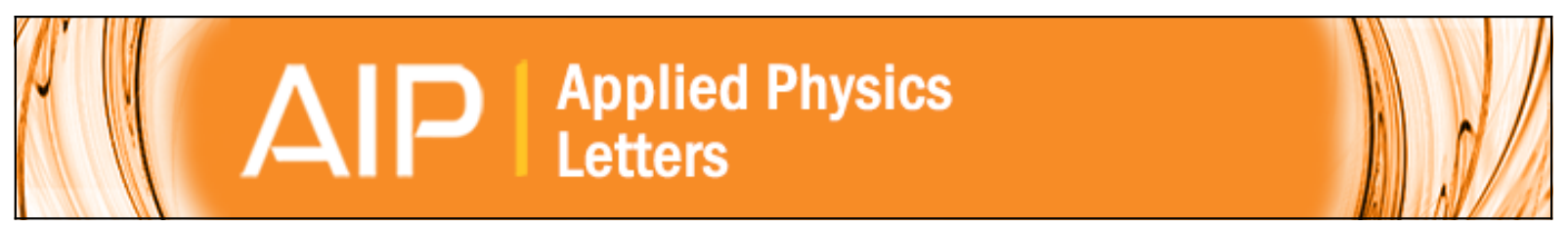

\title{
Characterization of the mechanical properties of HL-1 cardiomyocytes with high throughput magnetic tweezers
}

La Chen, Vanessa Maybeck, Andreas Offenhäusser, and Hans-Joachim Krause

Citation: Applied Physics Letters 107, 053703 (2015); doi: 10.1063/1.4928322

View online: http://dx.doi.org/10.1063/1.4928322

View Table of Contents: http://scitation.aip.org/content/aip/journal/apl/107/5?ver=pdfcov

Published by the AIP Publishing

\section{Articles you may be interested in}

High-throughput sorting of drops in microfluidic chips using electric capacitance

Biomicrofluidics 9, 044116 (2015); 10.1063/1.4928452

Magnetic approaches to study collective three-dimensional cell mechanics in long-term cultures (invited)

J. Appl. Phys. 115, 172616 (2014); 10.1063/1.4870918

Nanomechanics of magnetically driven cellular endocytosis

Appl. Phys. Lett. 99, 183701 (2011); 10.1063/1.3656020

High throughput system for magnetic manipulation of cells, polymers, and biomaterials

Rev. Sci. Instrum. 79, 083707 (2008); 10.1063/1.2976156

Magnetic tweezers for intracellular applications

Rev. Sci. Instrum. 74, 4158 (2003); 10.1063/1.1599066

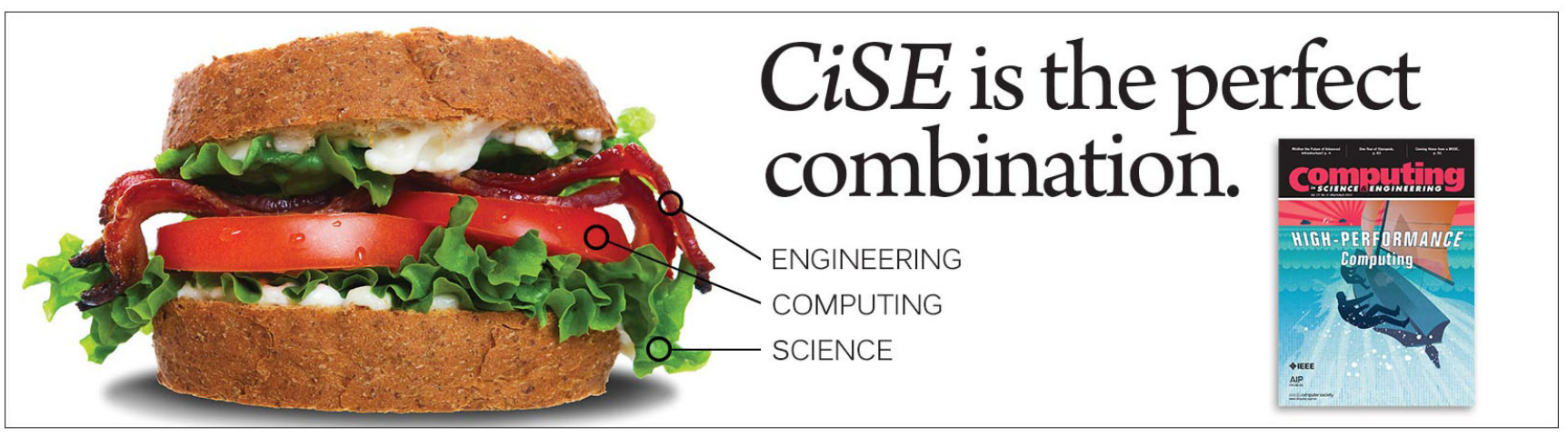




\title{
Characterization of the mechanical properties of HL-1 cardiomyocytes with high throughput magnetic tweezers
}

\author{
La Chen, Vanessa Maybeck, Andreas Offenhäusser, and Hans-Joachim Krause ${ }^{\text {a) }}$ \\ Institute of Bioelectronics (ICS-8/PGI-8), Forschungszentrum Jülich GmbH, 52425 Jülich, Germany
}

(Received 12 June 2015; accepted 29 July 2015; published online 7 August 2015)

\begin{abstract}
We characterized the mechanical properties of cardiomyocyte-like HL-1 cells using our recently developed multi-pole magnetic tweezers. With the optimized design, both high force and high throughput are achieved at the same time. Force up to $100 \mathrm{pN}$ can be applied on a $1 \mu \mathrm{m}$ diameter superparamagnetic bead in a workspace with $60 \mu \mathrm{m}$ radius, which is encircled symmetrically by 3 sharp magnetic tips. By adjusting the coil currents, both the strength and direction of force can be controlled. The result shows that both viscosity and shear elastic modulus of HL-1 cells exhibit an approximately log-normal distribution. The cells became stiffer as they matured, consistent with a transition from proliferating cells to contractile muscle tissue. Moreover, the mechanical properties of HL-1 cells show high heterogeneity, which agrees well with their physiological structure. (C) 2015 AIP Publishing LLC. [http://dx.doi.org/10.1063/1.4928322]
\end{abstract}

Both the mechanical properties of cells and their response to mechanical signals usually play an important role in various biological processes, such as differentiation, motility, adhesion, and mechanical signal sensing. ${ }^{1-4}$ In the last decades, a variety of microrheology techniques have been developed to actively or passively investigate the mechanical properties of cells. ${ }^{4,5}$ Due to their simple architecture and the specific properties of magnetic micro- and nanoparticle probes, magnetic tweezers (MTs) have been increasingly applied in studies of single molecules and of cell biophysics. ${ }^{6-9}$ It is well known in biology that statistical significance can only be achieved by multi-sample measurements because of large inherent variation from cell to cell. So, high throughput experimental setups are always favorable. Based on the developed inverse force model, our recently reported multi-pole magnetic tweezers combine high force output, good maneuverability due to active feedback control, and a large workspace. ${ }^{10}$ This allows us to address multiple cells in a single sample—reducing sample to sample variability and increasing throughput.

It was well established that the cytoskeleton in cardiomyocytes plays a pivotal role in sensing mechanical stress, mediating structural remodeling, and functional response in both physiological growth and pathological stimuli. ${ }^{11}$ The HL-1 cell line of cardiomyocyte-like cells, derived from a mouse atrial cardiomyocyte tumor persists in culture, yet matures to exhibit the essential characteristics of adult cardiomyocytes such as spontaneous electric activity and mechanical contraction upon reaching confluency. ${ }^{12}$ It has been used for studies of structure, function, and pathological conditions of cardiac muscle cells. ${ }^{13}$ In this work, we use our improved MT to study the mechanical properties of HL-1 cells.

Our magnetic tweezers consist of a main hexapole yoke and a specific fluidic cell with magnetic tips. The main yoke parts and the tips were laser-cut to the designed geometry (manufactured by SEKELS GmbH, Germany). As the magnetomotive source, the hexapole yoke is made of FeNi alloy

\footnotetext{
${ }^{\text {a) }}$ Author to whom correspondence should be addressed. Electronic mail: h.-j.krause@fz-juelich.de.
}

(PERMENORM $^{\circledR} 5000 \mathrm{H} 2$, Vacuumschmelze GmbH \& Co. $\mathrm{KG}$, Germany), which has a high permeability and a low coercivity. In order to generate a high force, the fluidic cell is equipped with 3 sharp magnetic tips made of high saturation magnetization $\mathrm{FeCo}-\mathrm{V}$ alloy $\left(\mathrm{VACOFLUX}^{\circledR}\right.$ 50, Vacuumschmelze GmbH \& Co. KG, Germany). In 3 poles MT, the maximum magnetic force can be achieved when the width of the tips equals the radius of the workspace. ${ }^{10}$ In case of thin tips, the practically achievable magnetic force is usually reduced by magnetic leakage flux bypassing along both sides of the tip. Only when the thickness of the tips is comparable to the distance between the tips, does this effect become negligible. In this work, the tips were cut from $100 \mu \mathrm{m}$ thick magnetic FeCo- $\mathrm{V}$ foil. Both the radius of the workspace and the width of the tips were set to $60 \mu \mathrm{m}$. Individual magnetic tips were aligned and fixed on a cover slip under the microscope with a small amount of slow setting glue. After drying of the glue, a glass ring was fixed on the cover slip with polydimethylsiloxane (PDMS, Sylgard 184, Dow Corning Corporation, USA). The magnetic force on a magnetic bead was calibrated by analyzing the viscous drag according to Stokes' formula. ${ }^{10}$ In the center of the workspace, forces higher than $100 \mathrm{pN}$ can be applied on a Dynabeads ${ }^{\circledR}$ MyOne Carboxylic Acid bead (Thermo Fisher Scientific, Inc., USA), which has a diameter of $1 \mu \mathrm{m}$ and a hydrophilic shell coating. In order to achieve good resolution, a high magnification water immersion objective (W Plan-Apochromat $63 \times / 1.0$ VIS-IR, Carl Zeiss AG, Germany) mounted on an upright microscope (Axio Scope.A1, Carl Zeiss AG, Germany) was used. The fluorescence and bright field images of the cells were captured using an ultra-low noise sCMOS camera (Zyla 5.5, Andor Technology Ltd., UK). The light from a light emitting diode (LED) cold-light source (CL 6000 LED, Carl Zeiss AG, Germany) was used to illuminate the workspace with a flexible light guide from the bottom of the fluidic cell. An illustration of this setup is shown in Fig. 1(a). The cells with magnetic particles were cultured on a small cover slip. In experiments, the cover slip was flipped upside down on the magnetic tips, as depicted in Fig. 1(a). The images acquired 
(a)

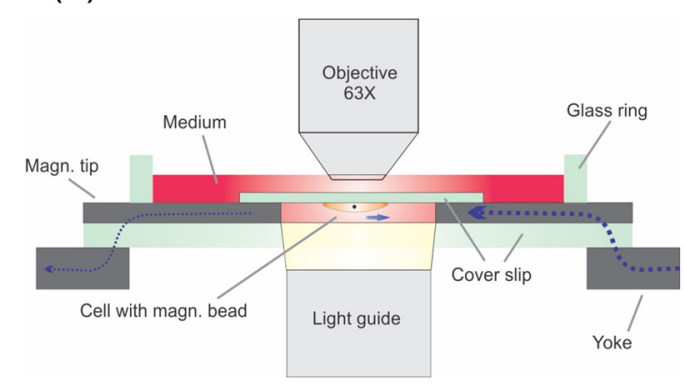

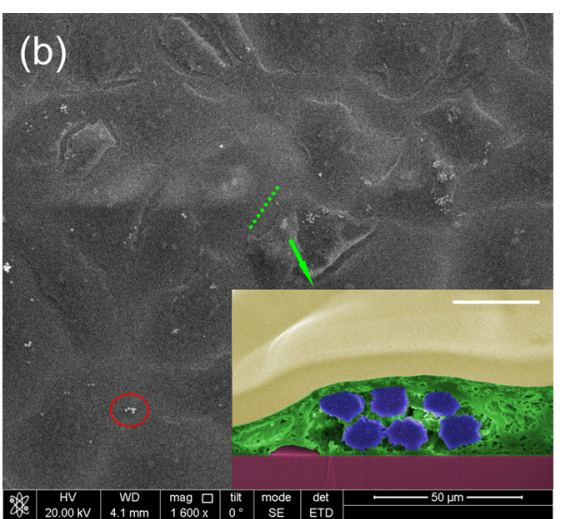

FIG. 1. (a) Schematic of our setup. The blue dashed lines illustrate the magnetic flux. (b) SEM image of HL-1 cells cultured on a $10 \times 10 \mathrm{~mm}^{2}$ silicon wafer substrate. The bright dots indicate magnetic beads. The beads in the red circle are located on the surface of the substrate in an area without cell coverage. The inset depicts a cross section after FIB cutting which was prepared parallel to the green dashed line. Pt deposition layer, cell material, magnetic bead, and Si substrate are shown, respectively, in yellow, green, blue, and red. The scale bar for inset figure is $2 \mu \mathrm{m}$. during the experiment were analyzed with a customized multi-bead tracking algorithm which combined the advantages of optical flow (OF) and cross-correlation (CC) algorithms. ${ }^{10,14}$ Each frame is first analyzed with OF. Then CC is carried out in a small local area based on the result of OF. Finally, the $\mathrm{CC}$ result is taken as the actual position of the bead, which is also used to update its initial position in the OF algorithm for the next frame. Thanks to the high magnification objective and the low noise camera, the achieved tracking resolution is less than $10 \mathrm{~nm}$, which agrees well with the findings of Ref. 15.

HL-1 cells were cultured on cover slips for 2-3 days. The nuclei of the living cells were stained with 4',6-diamidino-2-phenylindole (DAPI). The magnetic beads were mixed in advance with cell medium, and kept at $4{ }^{\circ} \mathrm{C}$. One night before mechanical measurement, the cells were fed with cell medium which includes both magnetic beads and DAPI. The final concentrations of beads and DAPI in medium were $7-12 \times 10^{6} / \mathrm{ml}$ and $10 \mu \mathrm{g} / \mathrm{ml}$, respectively. Before the experiment, the cover slip with cells was rinsed with phosphate buffered saline $(1 \times \mathrm{PBS})$. The fluidic cell was filled with $0.3 \mathrm{ml}$ PBS.

With the amide bonding reaction of carboxylic acid, the small beads bound to proteins and were easily endocytosed by the cells in culture. The real position of the beads was checked by focused ion beam-scanning electron microscope (FIBSEM). For this experiment, cells were cultured on a silicon substrate under the same conditions as mentioned before. (a)

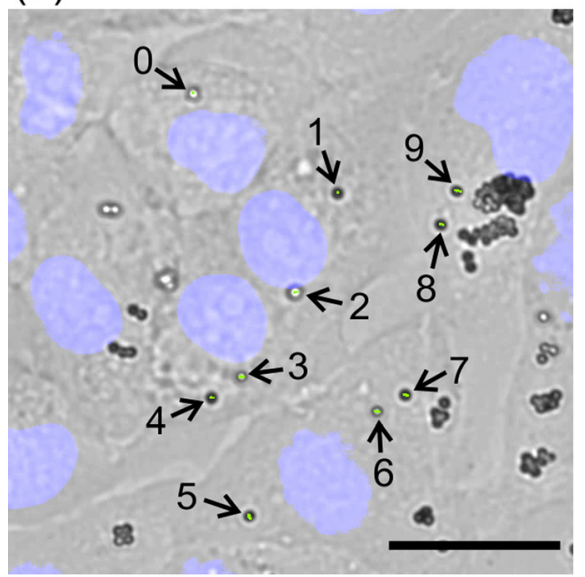

(c)

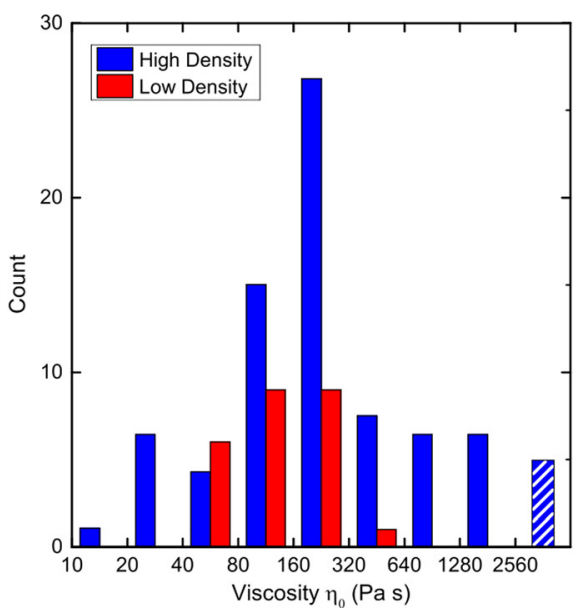

(b)

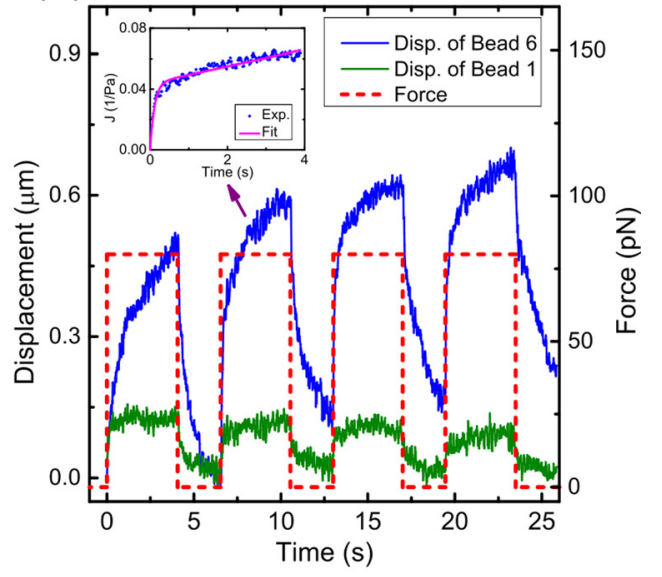

(d)

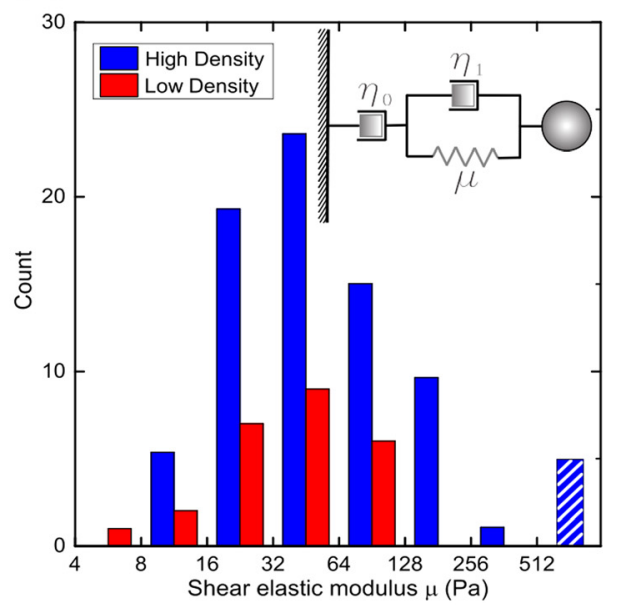

FIG. 2. (a) Multi cells measurement. The blue spots represent the cell nuclei stained with DAPI. The scale bar is $20 \mu \mathrm{m}$. (b) Displacement responses of beads 6 and 1 according to the applied force sequence. The inset depicts the second spike response of bead 6 and its fitting to the mechanical model, Eq. (1). (c) Viscosity $\eta_{0}$ distribution of 99 measured beads. The 5 beads out of range are indicated by the hatched bar. (d) Shear elastic modulus $\mu$ distribution of 99 measured beads. The 5 beads out of range are indicated by the hatched bar. The inset shows the adopted mechanical model. 
Then the cells were chemically fixed with $3.2 \%$ glutaraldehyde in PBS and dehydrated via ultrapure ethanol. In order to preserve the cell morphology, the ethanol in the samples was exchanged with $\mathrm{CO}_{2}$ via the critical point drying method. A sample with high density cell culture is depicted in Fig. 1(b). The beads' positions can be confirmed roughly using different electron acceleration voltages. For example, the beads on the surface are brighter, and the beads in cells are only visible under high energy electrons. In order to verify this finding, FIB cutting was carried out. As shown in the inset of Fig. 1(b), 3 beads piled above 3 other beads are in the cortex region of the cell. However, there were also some beads bound on the surface of the cells. And because of the activated surface of the beads, a few beads adhered on the substrate with the help of the cell medium (marked with a red circle in Fig. 1(b)), which can be distinguished by the response time of bead to the applied force. The bead bound on the substrate responds faster than the resolution of our optical detection.

Fig. 2(a) depicts a light microscope image of a measured sample. Only single beads were analyzed, bead clusters were ignored. In order to avoid possible interference from the neighboring bead clusters, bead 8 and bead 9 were also not included in the result. The responses of beads 6 and 1 are shown in Fig. 2(b). There are usually some "slips" in the first spikes which may come from the loose linkage between the bead and the cytoskeleton. ${ }^{16}$ Therefore, only the second or third spikes were analyzed in data processing. The response of the bead was fit by a simple mechanical model that consists of a Voigt body and a dashpot in series as shown in the inset figure in Fig. 2(d). The bead creep compliance can be described with ${ }^{17,18}$

$$
J(t)=g \frac{d(t)}{F(t)}=\frac{1-e^{-t / \tau}}{\mu}+\frac{t}{\eta_{0}} .
$$

Here, $\mathrm{g}=6 \pi r$ is a geometric factor related to the radius $r$ of the bead and $\tau=\eta_{1} / \mu$ is a relaxation time, depending on the Voigt body's viscosity $\eta_{1}$ and on the spring constant, the shear elastic modulus $\mu$. A total of 99 beads were analyzed. Both viscosity $\left(\eta_{0}\right)$ and shear elastic modulus $(\mu)$ show an approximately log-normal distribution, as seen in Figs. 2(c) and 2(d). This may derive from the high heterogeneity and intrinsic large variation of cell cytoskeletons, which were also widely observed in other rheology experiments. ${ }^{19,20} \mathrm{It}$ was found that the 69 out of 99 beads that were taken up in high cell density cultures ( $>80 \%$ coverage) encountered stiffer mechanical properties, i.e., a geometric mean viscosity of $\eta_{0}=209.83 \pm 3.01 \mathrm{~Pa} \mathrm{~s}$, a geometric mean shear elastic modulus of $\mu=48.33 \pm 2.2 \mathrm{~Pa}$, and a relaxation time of $\tau=0.171 \pm 0.157 \mathrm{~s}$. Moreover, 5 of the beads were found to be out of measurement range, of which the displacements were too small. This could be because the stiffnesses were too high or the particles were bound on the surface of the cell. In contrast, the other 25 beads that were found in less dense cell cultures $\left(<50 \%\right.$ coverage) exhibited $\eta_{0}=136.20$ $\pm 1.81 \mathrm{~Pa} \mathrm{~s}, \mu=35.64 \pm 2.05 \mathrm{~Pa}$, and $\tau=0.247 \pm 0.152 \mathrm{~s}$. Student's t-test was used to test for statistically significant differences in the parameters. The significant (probability of the difference occurring by chance $>0.9824$ ) observed stiffness increase with higher confluence probably originates from the cytoskeleton architecture remodeling and intracellular prestress reinforcing after cell-cell adhesions. ${ }^{4,11}$ However, our resultant mechanical parameter values such as elastic modulus are much lower than the results obtained in primary cardiac myocytes measured by atomic force microscopy. ${ }^{21,22} \mathrm{We}$ assume this difference may be due to the following facts: (1) these two methods actually characterize different cell regions which have different stiffnesses, according to Ref. 16. (2) The mechanical measurements are usually strongly dependent on how the mechanical model describes the linkage between the probe and cell cytoskeleton. ${ }^{4}$ (3) The cytoskeleton structure of HL-1 cells is different from the adult primary cells.

With the help of good maneuverability in the multi-pole MT, we also observed obvious heterogeneity of cell mechanical properties. As shown in Fig. 3(a), when the bead was actuated towards the nucleus, the response became stiffer. This effect can be interpreted by a denser actin meshwork and stress fibers close to the nuclei. ${ }^{16,23}$ Besides, there is an active response along the y direction. Unlike the regularly striated morphology of muscle tissue in vivo, the individual HL-1 cells cultured on glass still have a more radially organized cytoskeletal structure. ${ }^{23}$ In the protruding part of the cell, the stress fibers usually align, by which the cytoskeleton could anchor tightly with extracellular matrix and contribute to the protrusion. As seen in Fig. 3(b), it is obvious that the (a)

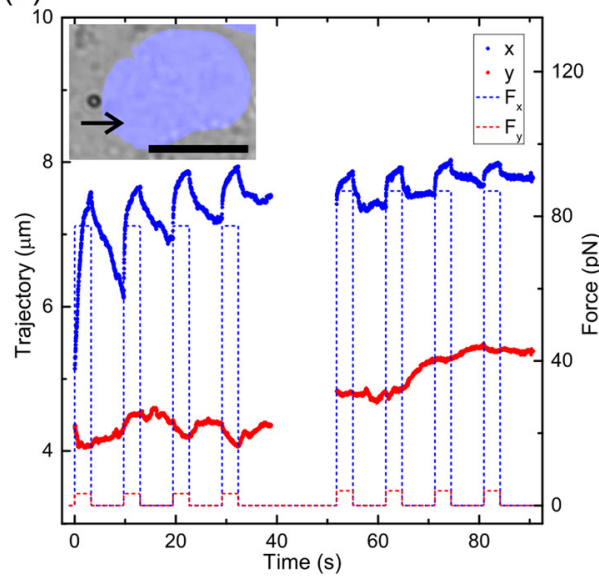

(b)

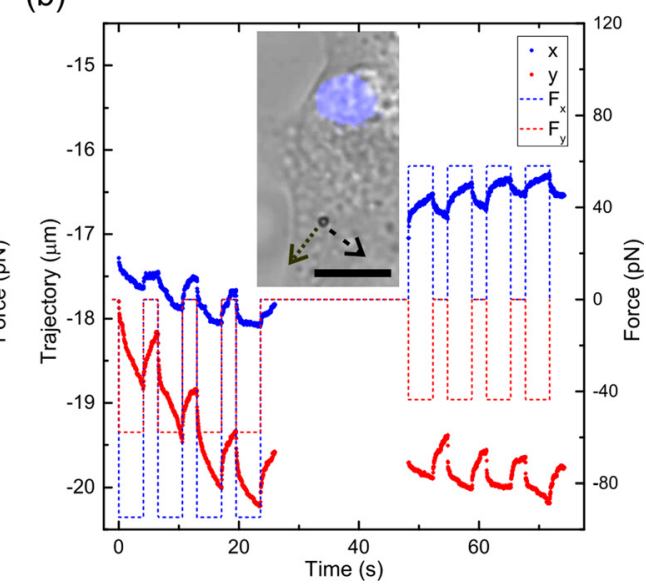

FIG. 3. Applied force sequence (dashed line) and observed particle trajectories (symbols) in horizontal $\mathrm{x}$ and vertical $y$ directions in case of (a) a particle close to the cell nucleus (the arrow shows the force direction) and (b) in the protrusion far away from the nucleus (the dotted arrow shows the force along the protrusion, the dashed arrow shows the force vertical to the protrusion). Both scale bars are $10 \mu \mathrm{m}$. 
elasticity is different in the directions parallel and perpendicular to the cell protrusion.

In conclusion, we characterized HL-1 cells' mechanical properties with high throughput, high force magnetic tweezers. Compared to commonly used single pole MTs, our multi-pole MT with a large workspace allows us to characterize many cells at a time. Thus, good statistics and efficiency can be easily achieved. Thanks to the good maneuverability in the multi-pole magnetic tweezers, a living cell can be probed in different directions to investigate cell heterogeneity without additional sample handling. The result shows that HL-1 cells have a log-normal distribution of their mechanical properties. The cell structure becomes stiffer with increasing confluence, i.e., when the cells begin to mature due to feedback from contact with neighboring cells. In addition, the mechanical properties of cells exhibit strong heterogeneity. The system can be enable comparative characterization of cell mechanics, for example, under genetic or pharmacological perturbations.

This work was performed in part at the Helmholtz Nanoelectronic Facility of Forschungszentrum Juelich. La Chen acknowledges support by the China Scholarship Council (No. 201206890062).

${ }^{1}$ G. Bao and S. Suresh, Nat. Mater. 2, 715 (2003).

${ }^{2}$ D. E. Discher, P. Janmey, and Y. Wang, Science 310, 1139 (2005).

${ }^{3}$ P. A. Janmey and C. A. McCulloch, Annu. Rev. Biomed. Eng. 9, 1 (2007).
${ }^{4}$ B. D. Hoffman and J. C. Crocker, Annu. Rev. Biomed. Eng. 11, 259 (2009).

${ }^{5}$ E. Moeendarbary and A. R. Harris, WIREs Syst. Biol. Med. 6, 371 (2014).

${ }^{6}$ I. D. Vlaminck and C. Dekker, Annu. Rev. Biophys. 41, 453 (2012).

${ }^{7}$ A. R. Bausch, F. Ziemann, A. A. Boulbitch, K. Jacobson, and E. Sackmann, Biophys. J. 75, 2038 (1998).

${ }^{8}$ P. Roca-Cusachs, A. D. Rio, E. Puklin-Faucher, N. C. Gauthier, N. Biais, and M. P. Sheetz, Proc. Natl. Acad. Sci. U. S. A. 110, E1361 (2013).

${ }^{9}$ A. H. B. D. Vries, B. E. Krenn, R. V. Driel, V. Subramaniam, and J. S. Kanger, Nano Lett. 7, 1424 (2007).

${ }^{10}$ L. Chen, A. Offenhäusser, and H.-J. Krause, Rev. Sci. Instrum. 86, 044701 (2015).

${ }^{11}$ M. L. McCain and K. K. Parker, Pflugers Arch.-Eur. J. Physiol. 462, 89 (2011).

${ }^{12}$ W. C. Claycomb, N. A. Lanson, Jr., B. S. Stallworth, D. B. Egeland, J. B. Delcarpio, A. Bahinski, and N. J. Izzo, Jr., Proc. Natl. Acad. Sci. U. S. A. 95, 2979 (1998).

${ }^{13}$ S. M. White, P. E. Constantin, and W. C. Claycomb, Am. J. Physiol. Heart Circ. Physiol. 286, H823 (2004).

${ }^{14}$ C. Gosse and V. Croquette, Biophys. J. 82, 3314 (2002).

${ }^{15}$ M. T. J. van Loenhout, J. W. J. Kerssemakers, I. De Vlaminck, and C. Dekker, Biophys. J. 102, 2362 (2012).

${ }^{16}$ C. M. Hale, S. X. Sun, and D. Wirtz, PLoS One 4, e7054 (2009).

${ }^{17}$ F. Ziemann, J. Rädler, and E. Sackmann, Biophys. J. 66, 2210 (1994).

${ }^{18}$ A. R. Bausch, W. Möller, and E. Sackmann, Biophys. J. 76, 573 (1999).

${ }^{19}$ N. Desprat, A. Richert, J. Simeon, and A. Asnacios, Biophys. J. 88, 2224 (2005).

${ }^{20}$ P. Cai, Y. Mizutani, M. Tsuchiya, J. M. Maloney, B. Fabry, K. J. V. Vliet, and T. Okajima, Biophys. J. 105, 1093 (2013).

${ }^{21}$ U. G. Hofmann, C. Rotsch, W. J. Parak, and M. Radmacher, J. Struct. Biol. 119, 84 (1997).

${ }^{22}$ S. C. Lieber, N. Aubry, J. Pain, G. Diaz, S. Kim, and S. F. Vatner, Am. J. Physiol. Heart Circ. Physiol. 287, H645 (2004).

${ }^{23}$ S. Pelloux, J. Robillard, R. Ferrera, A. Bilbaut, C. Ojeda, V. Saks, M. Ovize, and Y. Tourneur, Prog. Biophys. Mol. Biol. 90, 270 (2006). 